\title{
Bibliografía especializada
}

La presente bibliografía, que recoge una selección de monografias, articulos y documentos publicados desde 1990, pretende ser una aproximación para el estudio de la politica cultural en sus distintos aspectos: marco jurídico, institucional, agentes implicados, iniciativa privada, planes estratégicos, desarrollo económico y social, abarcando desde el ámbito internacional hasta el local, con especial atención al patrimonio cultural andaluz.

Los títulos marcados con asterisco $\left(^{*}\right)$ se encuentran disponibles para su consulta en la Biblioteca del IAPH. El resto de las referencias se han localizado en diversas fuentes de información (base de datos bibliográficas UNESBIB (UNESCO), Bibliografia Nacional, CSIC, Catálogo Colectivo Rebiun en Línea, Catálogo en línea del IPHE).

ABAD LICERAS, José María. Administraciones locales y patrimonio histórico. Madrid : Montecorvo, 2003

ABAD LICERAS, José María. Patrimonio histórico y jurisprudencia (1930-2003) [Recurso electrónico] .[Madrid : América Ibérica, 2003] *

ABAD LICERAS, José María. Propuestas para una nueva política de fomento del patrimonio histórico español : [ Salón Europeo del Arte de la Restauración, Rehabilitación y Conservación del Patrimonio Cultural. I Congreso Iberoamericano del Patrimonio Cultural : 29, 30 de noviembre y 1 de diciembre de 2001]. Madrid : América Ibérica, [2001] *

ABELLO TRUJILLO, Ignacio; ZUBIRÍA S., Sergio de; SÁNCHEZ F., Silvio. Cultura: teorías y gestión .1 1 $^{2}$ ed., reimp. San Juan de Pasto [Colombia] : Unariño, 1999 *

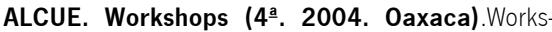
hops ALCUE Science \& Technology. Vol. 4, Cultural Heritage. Madrid : Consejo Superior de Investigaciones Científicas, Instituto de Automática Industrial, 2002 *

ALONSO HIERRO, Juan. El patrimonio histórico desde una perspectiva económica (una primera aproximación al gasto en conservación, restauración y rehabilitación del patrimonio histórico español). Madrid Fundación Caja Madrid, 2001 *

ALONSO IBAÑEZ, Rosario. Un futuro para la memoria : sobre la administración y el disfrute del patrimonio histórico español. Madrid : Visor, 2000 *

ÁLVAREZ ÁLVAREZ, José Luis. Los museos en la Ley de Patrimonio Histórico y en el Estado de las Autonomías. En Museos y la conservación del patrimonio. Madrid : Fundación BBVA : Antonio Machado Libros, D. L. 2001, pp. 41- 58
ÁLVAREZ ÁLVAREZ, José Luis. Estudios sobre el Patrimonio Histórico Español y la Ley de 25 de Junio de 1985. Madrid : Civitas, 1989 *

ÁLVAREZ ÁLVAREZ, José Luis. Sociedad, Estado y Patrimonio Cultural. Madrid : Espasa-Calpe, 1992 *

ANDALUCíA [Leyes, etc. de patrimonio histórico] Régimen jurídico del Patrimonio Histórico en Andalu-

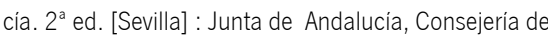
Cultura, D.L. $1997^{*}$

AYUDAS europeas a la cultura. Luxemburgo: Oficina de Publicaciones Oficiales de las Comunidades Europeas, 2002.

BALLART HERNÁNDEZ, Josep; JUAN I TRESSERRAS, Jordi. Gestión del patrimonio cultural. Barcelona : Ariel, 2001 *

BANÚS, E. Some remarks about the Cultural Policy of the European Union. En The Cultural Identity of Central Europe. Zagreb : Svob- Dokic, 1997

BARRAGÁN JANÉ, Montserrat. Sistema Andaluz de Museos y Registro de Museos en Andalucia. PH: Boletín del Instituto Andaluz de Patrimonio Histórico, 8 (34), 2001, pp. 159-165 *

BARRERA, David. Nuevas vias de financiación de proyectos patrimoniales. $\mathrm{PH}$ : Boletín del Instituto Andaluz del Patrimonio Histórico, n² 2, Diciembre 1998, pp. $158-162$ *

BARRERO RODRÍGUEZ, Concepción. La situación del patrimonio histórico en Andalucía : Un balance en el decimoquinto aniversario del Estatuto de Autonomía. RAAP, n² 27, 1996

BARRERO RODRÍGUEZ, Concepción. Patrimonio Cultural y organización administrativa. RAAP, n² 21, 1995.

BASES para una carta sobre Patrimonio y Desarrollo en Andalucía. [Sevilla] : Instituto Andaluz de Patrimonio Histórico, 1996 *

BASSAND, M. et BASTERRECHEA, L. An analytic review of the Report of the World Commission on Culture and Development. Paris : UNESCO, 1996

BATLLE, Albert. Espanya : les diputacions provin cials: una administració Intermèdia heterogènia. En L'Administració local i regional en l'espai cultural europeu: el rol de les administracions locals intermédies en la política cultural.[Barcelona] : Institut de Ciéncies Polítiques i Socials, 1999, pp. 24-46

BIBLIOGRAFÍA polítiques culturals [Literatura gris] : Interacció ‘96. Barcelona : Centre de Documentació del Centre d'Estudis i Recursos Culturals 1996 *
BIZKAIKO Ondare Historikoaren Ihardunaren Hamar urte $=$ Diez años de Gestión del Patrimonio Histórico de Bizkaia. [Bilbao] : Bizkaiko Foru Aldundia $=$ Diputación Foral de Bizkaia D.L. 1997 *

BONET, Lluís; CASTAÑER, Xavier; FONT, Joseph (eds.). Gestión de proyectos culturales : análisis de casos. $1^{\circ}$ ed. Barcelona : Ariel, 2001 (Ariel Practicum) *

BOORSMA, Peter B., HEMEL, Annemoon van, WIELEN, Niki van der (eds.) Privatisation and culture : experiences in the arts, heritage and cultural industries in Europe. Kluwer Academic Publishers, 1999

BOUZADA FERNÁNDEZ, Xan (ed.). Politicas culturales y sociedad democrática. Barcelona AESCA, 1999 [i.e. 2000]

BUSTAMANTE, Enrique (coord.); ÁLVAREZ MONZONCILLO, José María...[et al.]. Comunicación y cultura en la era digital : industrias, mercados y diversidad en España. Barcelona : Gedisa, 2002 *

CAETANO, Gerardo. Politicas culturales y desarrollo social: Algunas notas para revisar conceptos. Ábaco, 32-33, 2002, pp. 41-48 *

CAMPILLO GARRIGÓS, Rosa. La gestión y el gestor del patrimonio cultural. [Murcia] : KR, 1998, (Colección Historia y patrimonio) *

CAMPILLO GARRIGÓS, Rosa. La gestión y el gestor del patrimonio cultural [Archivo de ordenador] $=$ Cultural heritage management. [S.I. : s.d.], [2001] *

CARAVACA BARROSO, Inmaculada; COLORADO CAMPOS, David; FERNÁNDEZ SALINAS, Víctor; PANEQUE SALGADO, Pilar; PUENTE ASUERO, Raúl; ROMERO MORAGAS, Carlos. Patrimonio Cultural, Territorio y Políticas Públicas: El caso de Andalucia. Revista de Estudios Regionales, (47), 1997, pp. $143-160$

CARAVACA BARROSO, Inmaculada; COLORADO CAMPOS, David; FERNÁNDEZ SALINAS, Víctor; PANEQUE SALGADO, Pilar; PUENTE ASUERO, Raúl; ROMERO MORAGAS, Carlos. El patrimonio cultural como factor de desarrollo en Andalucía. PH: Boletín del Instituto Andaluz de Patrimonio Histórico, 5 (20), 1997, pp.87-97 *

CASA MARTínEZ, Carlos de la . Mecenazgo cultural y patrimonio histórico. Mercurio: Revista de Economía y Empresa, 1, 1997, pp.223- 248

CASTILLO OREJA, Miguel A. Los institutos de Patrimonio Histórico como organismos dependientes de las administraciones públicas. $\mathrm{PH}$ : Boletín del Instituto Andaluz del Patrimonio Histórico, $n^{\circ} 37$, diciembre 2001, pp. 156-161 * 
CóDIGO del patrimonio cultural de la Iglesia. Madrid : Edice, 2001 *

CONGRESO ANABAD (6‥1996.Murcia). Archivos, Bibliotecas, Centros de Documentación y Museos en la España de la Autonomias: actas del VI Congreso de ANABAD. [Murcia] : ANABAD-Murcia : Consejeria de Cultura y Educación, 1997 *

CURSO “DISEÑO Y EVALUACIÓN DE PROYECTOS CULTURALES" (2002. Málaga). Diseño y evaluación de proyectos culturales [Literatura gris]: [documentación del curso celebrado en Málaga del 4 al 8 de noviembre de 2002]. [Sevilla: IAPH, 2002] *

D'ANGELO, Mario. Cultural policies in Europe: regions and cultural descentralisation. Strasbourg: Council of Europe Publishing, 2000. (Training series)

DEBATE (1997 b). Debate: ordenación y coordinación de la realidad museistica. Administraciones autonómicas y museos, hacia un modelo racional de gestión. Santiago de Compostela, Dirección Xeral de Patrimonio Cultural, 1997. pp. 189-220

DIRECTORIO de Fundaciones 2000: protectorado de fundaciones culturales, docentes, de investigación y deportivas. [Madrid]: Ministerio de Educación y Cultura, Secretaría General Técnica, Centro de Publicaciones, D.L. 1999

DOS mil3 estrategias : proceso de evaluación institucional de la política cultural de Puerto Real. Cádiz : Universidad de Cádiz, Vicerrectorado de Extensión Universitaria ; Puerto Real : Ayuntamiento de Puerto Real, 2003 *

EI PATRIMONIO cultural [Número monográfico de la revista: Política y sociedad]. Enero-abril 1998, $n^{\circ}$ 27. Madrid: Facultad de Ciencias Politicas y Sociologia, Universidad Complutense, 1998 *

El SECTOR cultural en España ante el proceso de integración europea. [Madrid : Ministerio de Cultura], D.L. 1992 *

ENCUENTRO DE FUNDACIONES CULTURALES (1‥ 2000. MÁLAGA). La memoria en marcha: I Encuentro de Fundaciones Culturales, 25-26 de mayo de 2000. [Málaga]: Centro Cultural de la Generación del 27,2000

ESPAÑA [Leyes, etc.]. Legislación cultural de los paises del Convenio Andrés Bello: [España]. Santafé de Bogotá: Convenio Andrés Bello, 2000 *

ESPAÑA. Ministerio de Educación y Cultura. Normativa de museos de titularidad estatal. [Madrid : Ministerio de Educación y Cultura, Subdirección General de Museos Estatales], D.L. 1996 *
ESTADÍSTICAS 2000 Museos y colecciones en España. Madrid: Ministerio de Educación, Cultura y Deporte, D.L. 2003

EUROPA, Iglesia y patrimonio cultural : textos internacionales. Madrid : Biblioteca de Autores Cristianos, 1996 *

FAVRE D' ARCIER, Bernard. Estrategias para una nueva gestión cultural : tres experiencias significativas : [Conferencias realizadas en el Centro Cultural Ricardo Rojas de la Universidad de Buenos Aires, Mayo-Junio de 1998]. $1^{\text {a }}$ ed. Buenos Aires : EUDEBA, 1999

FEILDEN, Bernard M.; JOKILEHTO, Jukka. Manual para el manejo de los sitios del Patrimonio Cultural Mundial. [Roma] : ICCROM, 2003

FERNÁNDEZ-BACA CASARES, Román. Diez años desde el Instituto Andaluz del Patrimonio Histórico. PH : Boletín del Instituto Andaluz del Patrimonio Histórico, $n^{\circ} 37$, diciembre 2001, pp. 148-155 *

FERNÁNDEZ-BACA CASARES, Román. El patrimonio Cultural, un recurso frágil. Cuadernos de Gestión Pública local, 2000, pp. 205-209

FERNÁNDEZ DE GATA SÁNCHEZ, Dionisio. El régimen jurídico de protección del Patrimonio Histórico en la legislación autonómica. Patrimonio Cultural y Derecho, 3, 1999, pp.33-85 *

FERNÁNDEZ MIRANDA, Manuel. Iglesia Católica y Patrimonio Histórico. Foro del Patrimonio Histórico. Madrid: Fundación Cultural Banesto, pp. 211-214

FERNÁNDEZ DE MUNIAIN LETAMENDIA, CarIos; BENZO SAINZ, Isabel. Régimen de distribución de competencias entre el Estado y las Comunidades Autónomas: cultura y lengua. Madrid: Ministerio para las Administraciones Públicas. Instituto Nacional de Administración Pública, 1993. (Informes y Documentos. Administraciones territoriales)

FERNÁNDEZ PRADO, Emiliano. Administraciones y Politicas Culturales: Equilibrios y ambigüedades. En Ábaco, 1995, n. 5, pp. 49-61 *

FERNÁNDEZ PRADO, Emiliano. La politica cultural : qué es y para qué sirve. Gijón : Trea, 1991 *

FERNÁNDEZ SOBRADO, José Manuel; ANTOLÍN IRIA, José Enrique. La construcción administrativa de la realidad cultural en España. Revista Internacional de Sociologia, 27, 2000, pp.105-124

FLORES COLLAZO, María Margarita. Cultura y gestión cultural : una bibliografia indispensable. Río Piedras (Puerto Rico) : Universidad de Puerto Rico, 2002
GAITE PASTOR, Jesús. El sistema de archivos de la Administración central española. Revista General de Información y Documentación, 9 (2), 1999, pp. 127-136

GARCíA CANCLINI, Néstor. Opciones de políticas culturales en el marco de la globalización, en Seminario Nuevos retos y estrategias de las politicas culturales frente a la globalización. Institut d"Estudis Catalans. Barcelona, 22-25 noviembre 2000. Universitat de Barcelona

GARCía CANCLINI, Néstor Seminar on Cultural Policies and Development. Brasilia 2002. Politicas culturais para o desenvolvimiento; uma base de dados para a cultura (2003). (Disponible en http://www.unesco.org.br/download/publi_online/M iolo\%20POLITICAS\%200K.pdf)

GARCíA FERNÁNDEZ, Javier. La protección jurídica del Patrimonio Cultural. Nuevas cuestiones y nuevos sujetos a los diez años de la Ley de Patrimonio Histórico Español. Patrimonio Cultural y Derecho, $\mathrm{n}^{\circ}$ 1 , pp. $53-73^{*}$

GARCÍA FERNÁNDEZ , Javier. El régimen jurídico de los archivos, bibliotecas y museos de titularidad estatal conforme a la Constitución Española. Patrimonio Cultural y Derecho, 3, 1999, pp. 179-199 *

GARCíA GRACIA, María Isabel. La evolución de la industria de la cultural y el ocio en España por comunidades autónomas: (1993-1997). Madrid: Fundación Autor, 2001. (Datautor)

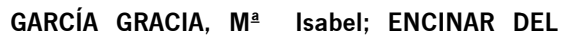
POZO, Mà Isabel; MUÑOZ PÉREZ, Félix-Fernando. La industria de la cultura y el ocio en España : su aportación al PIB. Madrid : Fundación Autor, D.L. 1997 *

GÉS, Marcel. Fondos y formas : recursos internacionales para proyectos culturales y artisticos. Barcelona : Interarts : Peninsula, 1999 *

GREFFE, Xavier. La gestion du patrimoine culturel. Paris : Anthropos, cop. 1999 *

GORDON, Christopher; MUNDY, Simon .European perspectives on cultural policy Culture, development and society series .Paris: UNESCO, 2001

GUÍA de experiencias de voluntariado cultural 98/99 : Andalucía. Sevilla : Consejería de Cultura de la Junta de Andalucia, Empresa Pública de Gestión de Programas Culturales, D.L. 2000 *

GUIDE pratique de l'intégration: les acteurs, les politiques et dispositifs publics, les sources d'information. Agence pour le développement des relations interculturelles (France). Paris: La Documentation française, 2002 
HÄBERLE, Peter . La protección constitucional y universal de los bienes culturales: un análisis comparativo. Revista Española de Derecho Constitucional, 54, 1998, pp. 11-38

HANDBOOK of cultural affairs in Europe $=\mathrm{Ma}$ nuel Européen des affaires culturelles = Manual de los Asuntos Culturales en Europa [...]; initiated by the Council of Europe (CDCC) with support from the Foreign Office of the Federal Republic of Germany

[et al.]. 3rd ed. Baden-Baden: Nomos Verlagsgesellschaft, cop. 2000 *

HERNÁNDEZ NÚÑEZ, Juan Carlos. Los instrumentos de tutela del patrimonio histórico español : sociedad y bienes culturales. [Cádiz] : Grupo Publicaciones de Sur, 1998 *

HERRERO PRIETO, Luis César. Economía de la cultura y el ocio. Nuevas posibilidades para la política económica regional : [Conferencia preparada para el Curso "Patrimonio Histórico y Economía", Universidad Internacional Alfonso VIII, Soria, 22 al 26 de julio de 1996]. Valladolid : Universidad de Valladolid, 1996

IBAÑEZ PICó, Marisol. El derecho a la cultura en el municipio español. Revista de Estudios de Administración Local y Autonómica, (282), 2000, pp.257-280

INDUSTRIAS y políticas culturales en España y País Vasco. Bilbao: Servicio Editorial Universidad del País Vasco, 1995 *

INFORME mundial sobre la cultura 2000-2001 : diversidad cultural, conflicto y pluralismo. Paris : Unesco ; Madrid : Mundi-Prensa, 2001 *

JIMÉNEZ CORNEJO, Encarna. ¿Cómo se protege el Patrimonio Histórico Andaluz?. Boletín Informativo del Instituto Andaluz del Patrimonio Histórico, n 6, 1994, pp. 22-24

JORNADAS “BIBLIOTECA PÚBLICA Y POLÍTICAS

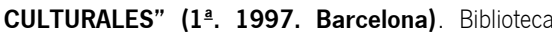
Pública y Políticas Culturales: Barcelona, abril 1997. Barcelona : Fundación Bertelsmann - España, 1997

JORNADAS SOBRE INICIATIVA PRIVADA Y SECTOR PÚBLICO EN LA GESTIÓN DE LA CULTURA (3a. 2001. Vitoria-Gasteiz). Cultura, desarrollo y territorio.. Vitoria-Gasteiz : Xabide, Gestión Cultural y Comunicación, 2001 *

JORNADAS SOBRE INICIATIVA PRIVADA Y SEC TOR PÚBLICO EN LA GESTIÓN DE LA CULTURA (1 ${ }^{a}$. 1997. Vitoria-Gasteiz). Público y privado en la gestión cultural. Vitoria-Gasteiz : Xabide, Gestión Cultural y Comunicación, 2000 *

KARIS : publicación bimestral de Interarts, Observatorio Europeo de Políticas Culturales Urbanas y Regionales. Barcelona : Asociación Interarts, 1997.
KOCKEL, Ullrich (ed.). Culture and economy: comtemporary perspectives. Aldershot, England; Burlington, VT : Ashgate, cop. 2002

KREBS, M.; SCHMIDT- HEBBEL, K. Patrimonio Cultural: Aspectos Económicos y Políticas de Protección. Perspectivas en Política Economía y Gestión 2(2) pp.207-45

La CULTURA andaluza en el umbral del siglo XXI Sevilla : Ayuntamiento de Sevilla, Servicio de Publicaciones, 2001 *

La GESTIÓN cultural : cursos : [1994-1995] [Murcia] : Caja Murcia, Obra Cultural, [1996]

Las CIFRAS de la cultura en España : estadísticas e indicadores. Edición 2002. Madrid: Ministerio de Educación, Cultura y Deporte, Subdirección General de Información y Publicaciones, 2002 *

LINDE PANIAGUA, Enrique...[ et. al.] Cultura y Desarrollo. Madrid: Ministerio de Cultura, Secretaría General Técnica, 1995. (Colección Análisis y Documentos; 7) *

LINDE PANIAGUA, Enrique. Nuevos y viejos retos de la politica cultural. En Puntos cardinales de la acción cultural en la España de nuestro tiempo. Madrid: Ministerio de Cultura, 1995, pp. 17-36 *

LINDE PANIAGUA, Enrique. Puntos cardinales de la acción cultural en la España de nuestro tiempo. Madrid Ministerio de Cultura, [1995] *

LÓPEZ BRAVO, Carlos. Los bienes culturales en e Derecho estatal y autonómico de España. Patrimonio Cultural y Derecho, 3, 1999, pp. 11-32 *

LÓPEZ DE AGUILETA, Iñaki. Cultura y ciudad: manual de política cultural municipal. Trea, 2002

MAPA de necesidades culturales : (MANECU). Madrid : Ministerio de Cultura, Secretaría General Técnica, 1995 *

MARTÍN Y PÉREZ DE NANCLARES, José. La atribución de competencias en materia de cultura (art. 128 TCE). Revista de Instituciones Europeas, 22 (1) 1995, pp. 171-189

MARTINELL, Alfons. Los agentes culturales ente los nuevos retos de la gestión cultural. Revista iberoamericana de Educación, 20, 1999, pp. 201-215

MARTINELL, Alfons. Nuevas competencias en la formación de gestores culturales ante el reto de la internacionalización. En Revista Pensar Iberoamérica $n^{\circ}$.2, octubre 2002 - enero 2003. Organización de Estados Iberoamericanos para la Educación, la Ciencia y la Cultura (OEI) [en línea]. Cuatrimestral (disponible en http://www.campus-oei.org/

pensariberoamerica/ric02a05.htm)

MEDIDAS de fomento y mecenazgo en materia de patrimonio cultural inmueble : experiencias comparadas en Europa. Madrid : Fundación de Casas Históricas y Singulares, 2002 *

MORENO NAVARRO, Isidoro. Cultura andaluza, patrimonio cultural y políticas de patrimonio. Demófilo: Revista de Cultura Tradicional de Andalucia,(1), 2002, pp. $71-87^{\text {* }}$

NÉGRIER, Enmanuel. Las políticas culturales en Francia y España: una aproximación nacional y local comparada. Institut de Ciéncies Politiques i Socials, 2003. (Working Papers ; 226). (Disponible en: http://www.ub.es/ cultural/politicasculturales/NEGRIER.pdf)

NORMATIVA sobre el patrimonio histórico cultural $\left[2^{\mathrm{a}}\right.$ ed. act.].Madrid : Ministerio de Educación y Cultura, Centro de Publicaciones, D.L. 1998 *

NUEVOS espacios para la cultura en Europa. Barcelona : Diputació de Barcelona : Milenio, 2000, (Interacció : materiales de política cultural; 3) *

OBJETIVOS y líneas de actuación de la política cultural (1983- 1992). Madrid: Ministerio de Cultura, [ 1993 ]

ORGANIZACIÓN DE ESTADOS IBEROAMERICANOS PARA LA EDUCACIÓN, LA CIENCIA Y LA CULTURA. Informes sobre los Sistemas Nacionales de Cultura [Recurso electrónico]. Madrid : Organización de Estados Iberoamericanos para la Educación, la Ciencia y la Cultura, D.L. 2002

PADRÓ, Jordi. Territorio y gestión creativa del patrimonio cultural y natural. En Ábaco, (34), 2002, pp. $55-60$ *

PATRIMONIO cultural en el contexto de Europa : textos, conceptos y concordancias. Madrid : Hispania Nostra : Boletín Oficial del Estado, 1999 *

PATRIMONIO Cultural y Derecho. Boletín Oficial del Estado; Fundación AENA; Fundación de los Ferrocarriles Españoles; Asociación Hispania Nostra. 1997. Madrid : Boletín Oficial del Estado [etc.], 1997 *

PATRIMONIO, museos y turismo cultural : claves para la gestión de un nuevo concepto de ocio : actas del curso celebrado en el marco de los Seminarios “Fons Mellaria” 1997 (Fuente Obejuna, Córdoba, 21-25 de julio). Córdoba : Servicio de Publicaciones de la Universidad de Córdoba, 1998 *

PENSAR la gestión cultural en Andalucía. [Huelva] : Asociación de Gestores Culturales de Andalucía, D.L. 2003 * 


\section{8}

\section{Debate}

e Investigación

Bibliografía especializada

PH48 - Abril 2004

PEREDA, Araceli. Mecenazgo y empresa pública. Aena Arte: revista cultural, 1, 1996, pp.56-59

PERROT-LANAUD, Monique. The Politics of culture Unesco, 2001 (UNESCO sources; 137) pp. 10-12

PICKARD, Robert. Patrimoine culturel europeén, v. II: Analyse des politiques et de la practique. Strasbourg: Council of Europe Publishing, 2002

PLAN Estratégico de Cultura 1997-2006: Planes Directores 1997-2000. [Toledo]: Junta de Comunidades de Castilla- La Mancha. Consejería de Educación y Cultura, D.L. 1998

PLAN GENERAL DE BIENES CULTURALES ANDALUCÍA 2000 [Archivo de ordenador]. Ed. electrónica realizada por www.laletradigital.com. Sevilla : Consejería de Cultura, D.L. 2000 *

POIRRIER, Philippe; VADELORGE, Loic. Pour une histoire des politiques du patrimoine. Paris: La Documentation française, 2003. Índice e Introducción disponibles en: http://www.culture.gouv.fr/culture/min/ comite-histoire/histoire-patrimoine/histoire_ patrimoine.htm

POLITIQUES culturelles en Europe: la problématique locale. Strasbourg : Council of Europe, 2000

POVEDA SÁNCHEZ, Vicente. Legislación y realidad: del patrimonio cultural español, estado, Comunidad, Municipio e Individuo. EN Congreso ciudades históricas vivas, ciudades del pasado: pervivencia y desarrollo, ponencias y comunicaciones, Mérida, 30, 31 de enero y 1 de febrero de 1997. Mérida : Editora Regional de Extremadura, 1997, pp. $45-47$ *

PRESAS BARROSO, Concepción. El patrimonio histórico eclesiástico en el Derecho español. Santiago: Universidad de Santiago de Compostela, 1994 *

PRIETO, María Gemma. Derecho Internacional y Bienes Culturales. Patrimonio Cultural y Derecho, 2, 1998, pp. 9-20 *

PROGRAMA Cultura 2000: convocatoria 2002 : información sobre resultados. [Madrid] : Ministerio de Educación, Cultura y Deporte, Subdirección General de Información y Publicaciones, D.L. 2002 *

QUESADA LÓPEZ, José Manuel. El patrimonio cultural y su gestión. Madrid: Universidad Nacional de Educación a Distancia, [ 2002]. (Textos de educación permanente)

REPERTORIO de textos internacionales del Patrimonio Cultural. Sevilla: Instituto Andaluz del Patrimonio Histórico; Granada : Comares, 2003, (Cuadernos; 14) (en prensa) *
ROMERO MORAGAS, Carlos. Nuevas políticas culturales. PH: Boletín del Instituto Andaluz de Patrimonio Histórico, Año 7, n. 28, 1999, pp. 38-42 *

ROSAS MANTECóN, Ana. La participación social en las nuevas políticas para el Patrimonio Cultural. En Patrimonio Etnológico. Nuevas perspectivas de estudio. [Sevilla] : Instituto Andaluz del Patrimonio Histórico [Granada] : Comares, 1999, pp. 34-51

RUBIO ARÓSTEGUI, Arturo. La Evaluación de las acciones de los ayuntamientos en el fomento de la cultura: una reflexión sobre la metodología. Karis, $n^{0} 7$ 1998, pp. 16-20

RUíZ GIL, José Antonio. Propuestas para la implantación de un modelo social de Gestión del Patrimonio Cultural en Andalucia. PH : Boletín del Instituto Andaluz de Patrimonio Histórico, n 14, Marzo 1996, pp. 28-32 *

RUIZ ROBLEDO, Agustín. La Constitución Cultural Española. Sevilla : Instituto Andaluz de Administración Pública, 1998

SÁNCHEZ, José A. (coord.). Práctica Artística y Politicas culturales. Murcia: Universidad de Murcia, 2003

SANTCOVSKY, Héctor. Los actores de la cultura. Prólogo de Joan Costa.1 ${ }^{a}$ ed. Barcelona : Hacer, 1995

SIMPOSIO INTERNACIONAL “LA GESTIÓN DEL PATRIMONIO CULTURAL” (2001 . Valladolid). La gestión del patrimonio cultural : la transmisión de un legado: [actas del Simposio Internacional, Valladolid, 24-26 de octubre de 2001]. Valladolid : Fundación del Patrimonio Histórico de Castilla y León, 2002 *

SEMINARIO “EL PATRIMONIO, PIEDRA ANGULAR DEL DESARROLLO ECONÓMICO Y LOCAL" (1997. Abbaye Royale de Fontevraud). El patrimonio, piedra angular del desarrollo económico y loca [Literatura gris] : Abbaye Royale de Fontevraud, 18, 19 20 juin 1997. 2 v. [S.I. : s.n.], [1997] *

SEMINARIO INTERNACIONAL “PATRIMONIO $Y$ PATROCINIO EMPRESARIAL, UNA PERSPECTIVA EUROPEA" (1998. Salamanca). Patrimonio y patrocinio empresarial, una perspectiva europea : [actas del] Seminario internacional. [Valladolid]: Fundación del Patrimonio Histórico de Castilla y León, D.L. 1999 *

TEXEIRA COELHO, José. Diccionario crítico de politica cultural: cultura e imaginario. Traducción de María Noemi Alfaro, Olga Correa, Ángeles Godinez y Leonardo Herrera. Jalisco (México): Secretaría de Cultura de Gobierno, 2000

The COUNCIL of Europe and cultural heritage 1954-2000: intergovernmental work: basic texts. Strasbourg : Council of Europa Publishing, cop. 2001
THROSBY, David. Economía y Cultura. Traducción de Cristina Piña y María Condor. $1^{a}$ ed. Madrid: Cambridge University Press, 2001

TURISMO cultural: el patrimonio histórico como fuente de riqueza: [actas del Simposio Internacional]. [Valladolid]: Fundación del Patrimonio Histórico de Castilla y León, D.L. 2000 *

UNESCO. Plan de Acción sobre Politicas Culturales para el Desarrollo. Conferencia Intergubernamental sobre Políticas Culturales para el Desarrollo. Estocolmo, 30 de marzo- 2 de abril de 1998. (Disponible en: http://www.unesco.org/culture/laws/stockholm/ html_sp/actionpl1.shtml)

URQUIJO GOITIA, José Ramón. Necesidades de I+D en el sector del Patrimonio Cultural. Nuevas demandas y tendencias de futuro. $\mathrm{PH}$ : Boletín del Instituto Andaluz del Patrimonio Histórico, n 32, Septiembre 2000, pp. $94-105$ *

VAQUER CABALLERÍA, Marcos. Estado y cultura: la función cultural de los poderes públicos en la constitución española. Madrid : Editorial Centro de Estudios Ramón Areces: Universidad Carlos III de Madrid, D.L. 1998

VISUALES GONZÁLEZ, Jesús; GUITART I AGELL, Joan; LISSAVETZSKY DÍEZ, Jaime. La administración cultural española. Las CCAA : gestión y patrimonio cultural. En Cultura y Desarrollo. Madrid: Ministerio de Cultura. Secretaria General Técnica, 1995, pp. $217-240$

ZUBIRÍA SAMPER, Sergio de; ABELLO TRUJILLO, Ignacio; TABARES, Marta. Conceptos básicos de administración y gestión cultural. Madrid : Organización de Estados Iberoamericanos para la Educación, la Ciencia y la Cultura, D.L. 2001 *
Isabel Guzmán Guzmán

Lorena Ortiz Lozano

Centro de Documentación del IAPH 